\title{
Percepção da imagem corporal em adolescentes e a relação com seu estado
}

\section{nutricional}

\author{
Perception of body image in adolescents and the relationship with their nutritional \\ Percepción de la imagen corporal en adolescentes y relación con su estado nutricional
}

Recebido: 23/12/2020 | Revisado: 25/12/2020 | Aceito: 29/12/2020 | Publicado: 03/01/2021

Lidianne de Sousa Ferreira

ORCID: https://orcid.org/0000-0003-2635-2306

Centro Universitário Fametro, Brasil

E-mail: sousalidianne2@gmail.com

Tainá Cunha Rodrigues

ORCID: https://orcid.org/0000-0003-2445-1458

Centro Universitário Fametro, Brasil

E-mail: tainaacunhaaa@gmail.com

Valéria Silva de Lima

ORCID: https://orcid.org/0000-0002-7130-5173

Centro Universitário Fametro, Brasil

E-mail: valerialimma.nutri@gmail.com

Alane Nogueira Bezerra

ORCID: https://orcid.org/0000-0003-0586-1881

Centro Universitário Fametro, Brasil

Centro Universitário Christus, Brasil

Universidade Federal do Ceará, Brasil

E-mail: alane.bezerra@ professor.unifametro.edu.br

Natasha Vasconcelos Albuquerque

ORCID: https://orcid.org/0000-0003-4267-1120

Universidade Federal do Ceará, Brasil

E-mail: natashava@hotmail.com

Camila Pinheiro Pereira

ORCID: https://orcid.org/0000-0002-5482-3665 Centro Universitário Fametro, Brasil

Universidade Estadual do Ceará, Brasil

E-mail: camicpp@gmail.com

\begin{abstract}
Resumo
A imagem corporal é criada a partir da assimilação individual que cada pessoa em sua singularidade cria de si mesma. $\mathrm{Na}$ adolescência identifica-se um aumento nas percepções e reflexões sobre a forma do corpo, podendo estar associada aos padrões de beleza impostos pela sociedade. Tais fatores podem favorecer ao aumento da obesidade e de transtornos alimentares nessa fase da vida. O presente estudo tem por objetivo avaliar a autopercepção da imagem corporal e o estado nutricional de adolescentes estudantes do ensino médio de uma escola pública de Fortaleza-CE. Trata-se de um estudo transversal, com abordagem quantitativa, que avaliou 104 adolescentes de ambos os sexos. Para a avaliação do estado nutricional foi aferido o peso e estatura para o cálculo do Índice de Massa Corporal (IMC), sendo este convertido em Escore-Z de IMC/idade. Para a análise da percepção de imagem corporal foi utilizada a Escala de Silhueta Corpórea (EFS). Verificou-se que em ambos os sexos, foi encontrada, uma frequência maior de eutrofia, sendo $75,95 \%$ no sexo feminino e $60 \%$ no sexo masculino. Em relação à satisfação e a insatisfação corporal, $31,48 \%$ do sexo feminino encontraram-se insatisfeitas com o corpo. No sexo masculino, $10 \%$ encontraram-se insatisfeitos. Os resultados encontrados mostraram um percentual de insatisfação representativo em ambos os sexos. Conclui-se que a alta frequência de insatisfação corporal observada no presente estudo traz como importância a necessidade de desenvolver políticas públicas, com o foco nessa problemática.
\end{abstract}

Palavras-chave: Autoimagem; Distorção da percepção; Transtornos alimentares; Adolescente.

\begin{abstract}
The body image is created from the individual assimilation that each person in his uniqueness creates from himself. In adolescence, an increase in perceptions and reflections on the shape of the body is identified, which may be associated with the standards of beauty imposed by society. Such factors may favor the increase in obesity and eating disorders at this stage of life. The present study aims to assess the self-perception of body image and the nutritional status of high school teenagers from a public school in Fortaleza-CE. This is a cross-sectional study, with a quantitative approach, which evaluated 104 adolescents of both genders. For the assessment of nutritional status, weight and height were measured to calculate the Body Mass Index (BMI), which was converted into a Z-score of BMI / age. For
\end{abstract}


the analysis of the perception of body image, the Body Silhouette Scale (EFS) was used. It was found that in both sexes, a higher frequency of eutrophy was found, $75.95 \%$ in females and $60 \%$ in males. In relation to body satisfaction and dissatisfaction, $31.48 \%$ of women were dissatisfied with their bodies. Among males, $10 \%$ were dissatisfied. The results found showed a representative percentage of dissatisfaction in both sexes. It is concluded that the high frequency of body dissatisfaction observed in the present study highlights the need to develop public policies, with a focus on this issue.

Keywords: Self image; Distortion of perception; Eating disorders; Teenager.

\section{Resumen}

La imagen corporal se crea a partir de la asimilación individual que cada persona en su singularidad crea de sí mismo. En la adolescencia se identifica un aumento de las percepciones y reflexiones sobre la forma del cuerpo, lo que puede estar asociado a los estándares de belleza impuestos por la sociedad. Tales factores pueden favorecer el aumento de la obesidad y los trastornos alimentarios en esta etapa de la vida. El presente estudio tiene como objetivo evaluar la autopercepción de la imagen corporal y el estado nutricional de adolescentes de secundaria de una escuela pública de Fortaleza-CE. Se trata de un estudio transversal, con enfoque cuantitativo, que evaluó a 104 adolescentes de ambos sexos. Para la evaluación del estado nutricional, se midieron el peso y la altura para calcular el Índice de Masa Corporal (IMC), que se convirtió en una puntuación Z de IMC / edad. Para el análisis de la percepción de la imagen corporal se utilizó la Escala de Silueta Corporal (EFS). Se encontró que en ambos sexos se encontró una mayor frecuencia de eutrofia, 75,95\% en mujeres y $60 \%$ en hombres. En relación a la satisfacción e insatisfacción corporal, el 31,48\% de las mujeres estaban insatisfechas con su cuerpo. Entre los hombres, el 10\% estaba insatisfecho. Los resultados encontrados mostraron un porcentaje representativo de insatisfacción en ambos sexos. Se concluye que la alta frecuencia de insatisfacción corporal observada en el presente estudio destaca la necesidad de desarrollar políticas públicas, con enfoque en este tema.

Palabras clave: Auto imagen; Distorsión de la percepción; Trastornos de la alimentación; Adolescente.

\section{Introdução}

A imagem corporal é um conceito multidimensional, que representa a percepção que o indivíduo tem com relação ao a seu corpo e pode ser influenciada pelas representações comportamentais da personalidade e os estados emocionais. Considerase que a imagem corporal é uma dimensão do autoconceito (Lopes, Mendes \& Sousa, 2017).

A formação da imagem corporal pode ser influenciada por diversos fatores, tais como: gênero, ciclo de vida, satisfação corporal, além da relação com o corpo, com os processos cognitivos, afetivos e sociais. O nível socioeconômico, as crenças, a mídia, a escola, os valores, as atitudes e a própria cultura também têm grande relevância na criação da imagem corporal (Gomes, 2015).

Observa-se uma elevada prevalência de insatisfação com a imagem corporal em adolescente, além de um aumento no número de casos de anorexia e bulimia nessa fase (Martins, Pelegrine, Matheus \& Petroski, 2010). A adolescência é um ciclo compreendido entre a infância e a idade adulta, sendo uma etapa na qual ocorrem mudanças biológicas e psicossociais. É uma fase em que surgem dúvidas, e criam-se valores e percepções mais críticas sobre a sociedade e de si mesmo (Dias, 2017).

$\mathrm{Na}$ adolescência ocorre uma intensificação na preocupação com a imagem corporal, uma vez que a sociedade impõe, de forma direta e indireta, alguns padrões de beleza tidos como requisitos favoráveis à aceitação na sociedade (Gomes, 2015). A exigência imposta pela mídia e pela sociedade na busca pelo corpo ideal exerce relevante influência sobre o modo como os sujeitos percebem a si mesmos diante do espelho. O desejo de alcançar os padrões de beleza atuais e, muitas vezes, a impossibilidade de torná-los realidade, produz insatisfação com a imagem corporal (Fontenele et al., 2019). Tais fatores podem levar a insatisfação corporal como um dos critérios diagnósticos dos transtornos alimentares (TAs) (Fortes, Almeida \& Ferreira, 2013).

Os Transtornos Alimentares são caracterizados como transtornos psiquiátricos, e os indivíduos acometidos são identificados por mostrarem desvio de padrão no comportamento alimentar e alteração da imagem corporal, que leva a um grave prejuízo à saúde. Geralmente, apresentam seus primeiros sintomas na infância e adolescência. Essa patologia teve um crescimento significativo, tendo maior prevalência na população feminina (Alvarenga, 2015). 
Os TAs são responsáveis por causar um conjunto de danos e complicações físicas e psicológicas, os principais prejuízos físicos incluem alterações fisiológicas, déficit no crescimento e alterações hormonais. Os problemas psicossociais de maior frequência são isolamento social e desenvolvimento de transtorno depressivo e de ansiedade, que aumenta o risco de suicídio (Carvalho, 2019).

Estudos apontam que a prevalência da anorexia nervosa (AN) é de $0,4 \%$ entre jovens do sexo feminino. A proporção entre o sexo masculino é de 1 entre 10 casos em relação ao sexo feminino. Entretanto, os dados da Associação Americana de Nutrição indicam que a prevalência para AN é de $0,3 \%$ nos homens e $0,9 \%$ nas mulheres. Verifica-se que os dados epidemiológicos de transtornos alimentares são limitados, pois tanto a AN quanto a bulimia nervosa têm baixa prevalência na população em geral. Todavia, essa escassez de dados pode ser justificada, pois a maioria dos pacientes não procuram ir em busca de tratamento (Silva \& Mura, 2016).

Devido a necessidade de investigar dados de que possam alertar a sociedade para o risco de desenvolvimento dos TAs, com uma maior ênfase aos adolescentes, o presente estudo teve como objetivo avaliar a autopercepção da imagem corporal e sua relação com o estado nutricional de adolescentes estudantes do ensino médio, de uma escola pública de Fortaleza - CE.

\section{Metodologia}

O presente estudo foi de natureza quantitativa com delineamento transversal, acerca da percepção da imagem corporal. O cálculo do tamanho da amostra ideal foi realizado através da fórmula de Santos (2018), obtendo-se o quantitativo estimado para uma população finita de 298 alunos. Foi realizado em uma escola municipal situada na cidade de Fortaleza, Ceará, no mês de outubro de 2018, nos turnos da manhã e tarde. Foram incluídos no estudo adolescentes com faixa etária de 14 a 18 anos, de ambos os sexos, regularmente matriculados do $1^{\circ}$ ao $3^{\circ}$ ano do ensino médio. Foram excluídos do estudo adolescentes grávidas, que apresentavam algum tipo de deficiência física.

Antes da realização da coleta, foram fornecidos aos pais ou responsáveis dos adolescentes participantes da pesquisa, os termos de Consentimento Livre e Esclarecido (TCLE) e Termo de assentimento livre e esclarecido (TALE) para realização da avaliação, na qual foi classificada a percepção da imagem corporal através da Escala de Silhueta Corpórea (EFS).

As informações sobre a percepção e insatisfação da imagem corporal dos adolescentes foram coletadas a partir da avaliação das EFS criada por Stunkard, Sorenson e Schlusinger (1983). Esse instrumento foi validado para a realidade brasileira por Fidelix et al. (2011). Para análise da insatisfação, a EFS foi apresentada aos adolescentes e os mesmos responderam a duas perguntas: 1. Qual a silhueta que melhor representa a sua aparência corporal atual (real); 2. Qual é a silhueta corporal que você gostaria de ter (ideal)? Foram classificados como satisfeitos, quando a variação entre a silhueta auto avaliada como real e como ideal fosse igual a zero e insatisfeita quando diferente de zero. Quando a diferença (real-ideal) foi positiva, foi classificada como uma insatisfação pelo desejo de reduzir a silhueta e, quando negativa, uma insatisfação pela vontade ser aumentada (Fidelix et al., 2011).

A autopercepção da imagem corporal foi obtida pela autoavaliação seguindo o método realizado por Madrigal-frits e colaboradores (1999) e adaptado por Branco e colaboradores (2006), no qual se estabelecem quatro categorias: baixo peso (1), eutrofia (2 a 5), sobrepeso (6 e 7) e obesidade (8 e 9).

Para a avaliação do estado nutricional foi utilizado o peso e estatura para o cálculo do Índice de Massa Corporal (IMC $=$ peso/altura ${ }^{2}$ ), que foi convertido em Escore-Z do índice IMC/Idade com os seguintes parâmetros de classificação. $<$ Escore-Z -3 (Magreza), $\geq$ Escore $-\mathrm{z}-1 \mathrm{e} \leq$ Escore $\mathrm{z}-+1$ (Eutrofia), $>$ Escore- $\mathrm{z}+1 \mathrm{e} \leq$ Escore $-\mathrm{z}+2$ (Sobrepeso) e $>$ Escore $-\mathrm{z}+3$ (Obesidade), utilizando a tabela de referência da World Health Organization (WHO, 2007). 
Os procedimentos desta pesquisa seguiram a resolução 466/12 do Conselho Nacional de Saúde, que dispões sobre as normas de pesquisas envolvendo seres humanos (BRASIL, 2012). Portanto, os dados foram coletados de forma ética e organizada, garantindo a segurança e sigilo no desenvolvimento dos questionários e das avaliações. O presente estudo foi submetido e aprovado pelo Comitê de Ética em Pesquisa da Faculdade Metropolitana da Grande Fortaleza, pelo número de aprovação 2.865 .262 .

A tabulação dos dados foi realizada através do Microsoft Office Excel versão 2016. A apresentação dos resultados ocorreu através de tabelas, por meio de frequências absolutas e relativas.

\section{Resultados}

O estudo foi realizado com 104 adolescentes do ensino médio de uma escola pública, sendo 51,92\% (n=54) do sexo feminino e 48,8\% (n=50) do sexo masculino, com idade média e desvio padrão. De acordo com os dados antropométricos obtidos (Tabela 1), foi possível observar, segundo o índice IMC/Idade, que foram de maior frequência nos adolescentes do sexo masculino e feminino a classificação de eutrofia.

Tabela 1 - Distribuição do estado nutricional com base no IMC dos adolescentes do ensino médio de uma escola pública da cidade de Fortaleza de acordo com o sexo. Fortaleza-Ce 2018.

\begin{tabular}{|c|c|c|c|c|c|}
\hline \multirow{2}{*}{ Sexo } & \multicolumn{5}{|c|}{ Estado nutricional } \\
\hline & Magreza & Eutrofia & Sobrepeso & Obesidade & Total \\
\hline & $\mathrm{n}(\%)$ & $\mathrm{n}(\%)$ & $\mathrm{n}(\%)$ & $\mathrm{n}(\%)$ & $\mathrm{n}$ \\
\hline Feminino & $1(1,85 \%)$ & $41(75,93 \%)$ & $10(18,52 \%)$ & $2(3,70 \%)$ & 54 \\
\hline Masculino & $7(14 \%)$ & $30(60 \%)$ & $10(20 \%)$ & $3(6 \%)$ & 50 \\
\hline
\end{tabular}

Fonte: Autores (2018).

Nas Tabelas 2 e 3, encontra-se a autopercepção reconhecida pelos adolescentes, de acordo com o sexo. 
Tabela 2 - Frequência de respostas que retratam a autopercepção da imagem corporal (reconhecida) na escala de silhuetas de adolescentes do sexo feminino. Fortaleza-Ce 2018.

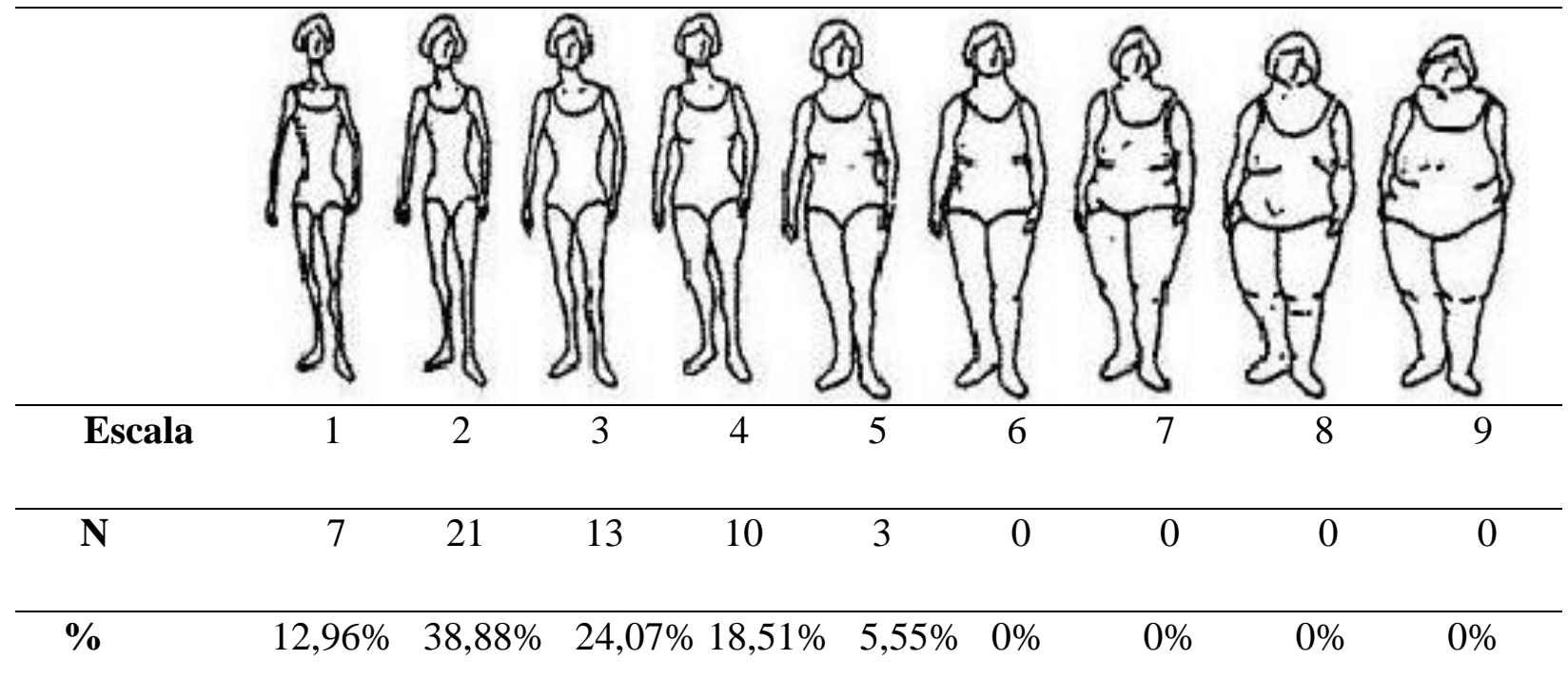

Fonte: Autores (2018)

Através da análise da Tabela 2, identificou-se que a maior frequência das adolescentes do sexo feminino $(38,88 \%)$ reconheceu possuir a silhueta 2 . Observou-se também que 38,88\% das 54 estudantes, identificaram-se eutróficas e nenhuma se identificou com a imagem de sobrepeso e obesidade, apesar de 3,70\% dessa amostra encontrar-se com obesidade. No sexo masculino, observou-se que $3060 \%$ encontraram-se eutróficos. Foi observada uma superestimação, pois a maioria deles percebem-se eutróficos, seguidos $1^{\circ}$ lugar $20 \%$ com sobrepeso.

Tabela 3 - Frequência de respostas que retratam a autopercepção da imagem corporal (reconhecida) na escala de silhuetas (sexo masculino). Fortaleza-Ce, 2018.

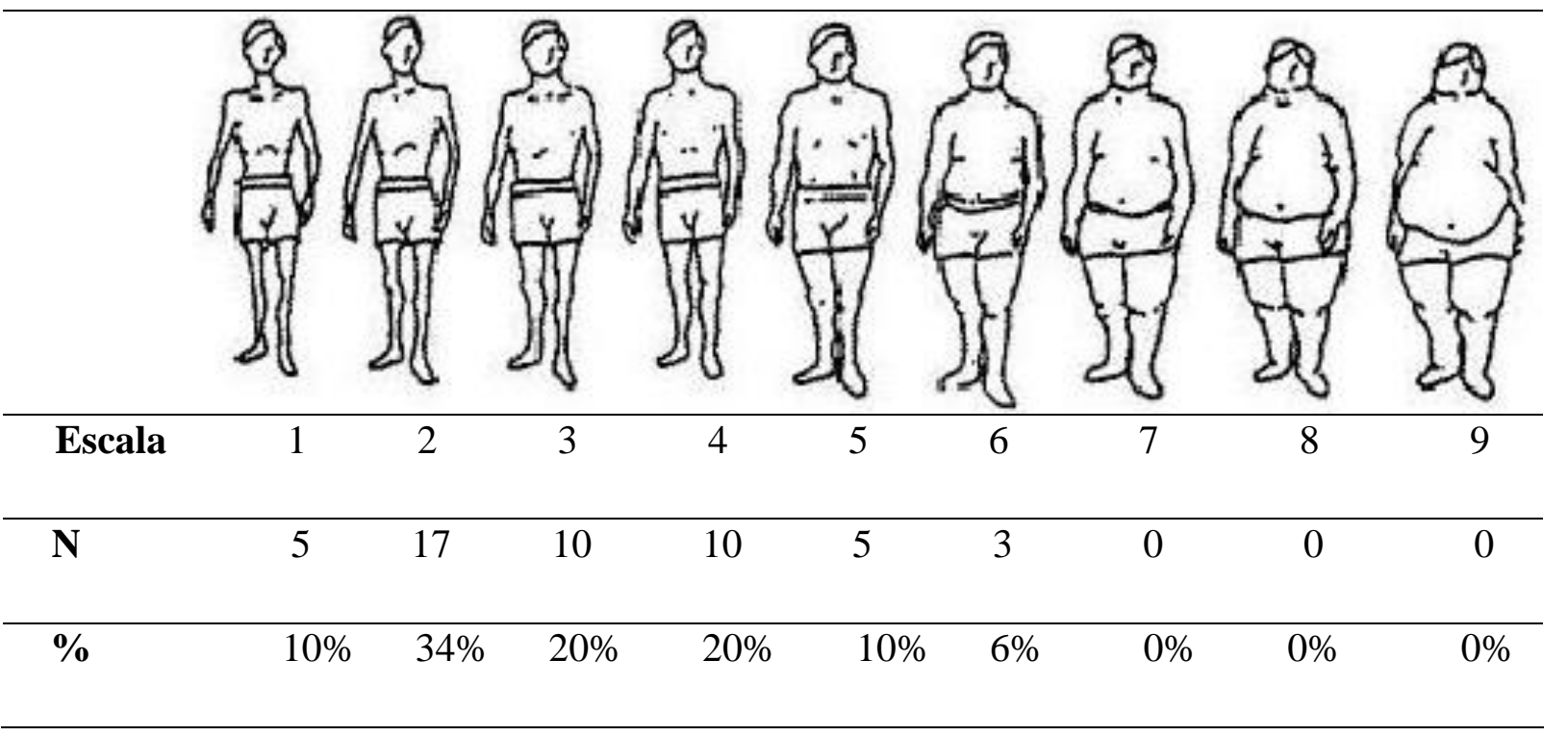

Fonte: Autores (2018). 
A relação da autopercepção da imagem com o estado nutricional dos adolescentes está apresentada na Tabela 4. Verificou-se que no sexo feminino foi referida mais vezes a imagem de número $2(55,5 \%)$, quando analisada a silhueta desejada entre os adolescentes de ambos os sexos.

Tabela 4 - Autopercepção da imagem e o estado nutricional dos adolescentes do ensino médio de uma escola pública da cidade de Fortaleza de acordo com o sexo. Fortaleza-Ce, 2018.

\begin{tabular}{|c|c|c|c|c|c|}
\hline \multirow{2}{*}{$\begin{array}{c}\text { Estado } \\
\text { Nutricional } \\
\text { Sexo }\end{array}$} & \multicolumn{5}{|c|}{ Autopercepção da imagem corporal } \\
\hline & $\begin{array}{l}\text { Silhueta } \\
\text { Magreza } \\
\text { N (\%) }\end{array}$ & $\begin{array}{l}\text { Silhueta } \\
\text { Eutrofia } \\
\text { N (\%) }\end{array}$ & $\begin{array}{c}\text { Silhueta } \\
\text { Sobrepeso } \\
\text { N (\%) }\end{array}$ & $\begin{array}{c}\text { Silhueta } \\
\text { Obesidade } \\
\text { N (\%) }\end{array}$ & $\begin{array}{c}\text { Total } \\
\text { N }(100 \%)\end{array}$ \\
\hline \multicolumn{6}{|l|}{ Baixo Peso } \\
\hline $\mathbf{M}$ & $2(28,57)$ & $5(71,43)$ & - & - & $7(6,73)$ \\
\hline $\mathbf{F}$ & $1(100,0)$ & - & - & - & $1(100,0)$ \\
\hline \multicolumn{6}{|l|}{ Eutrofia } \\
\hline $\mathbf{M}$ & $3(10,0)$ & $26(86,67)$ & $1(3,33)$ & - & $30(28,85)$ \\
\hline $\mathbf{F}$ & $6(14,63)$ & $35(85,37)$ & - & - & $41(39,42)$ \\
\hline \multicolumn{6}{|l|}{ Sobrepeso } \\
\hline M & - & $10(100,0)$ & - & - & $10(9,61)$ \\
\hline $\mathbf{F}$ & - & $10(100,0)$ & - & - & $10(9,61)$ \\
\hline \multicolumn{6}{|l|}{ Obesidade } \\
\hline $\mathbf{M}$ & - & $1(33,33)$ & $2(66,67)$ & - & $3(2,88)$ \\
\hline $\mathbf{F}$ & - & $2(100,0)$ & - & - & $2(1,92)$ \\
\hline \multicolumn{6}{|l|}{ Total } \\
\hline $\mathbf{M}$ & $5(10,0)$ & $42(84,0)$ & $3(6,0)$ & - & $50(100)$ \\
\hline $\mathbf{F}$ & $7(12,96)$ & $47(87,04)$ & - & - & $54(100)$ \\
\hline
\end{tabular}

M: masculino; F: Feminino. Fonte: Autores (2018).

Com base na análise da Tabela 4, verificou-se que o sexo masculino e feminino se encontrava com a percepção real de sua condição distorcida, na qual ocorreu em ambos os sexos uma subestimação. Das 41 adolescentes do sexo feminino, que se encontravam eutróficas, 14,63\% idenficaram-se com magreza, e dentre as 10 classificadas com sobrepeso e com obesidade, ambas se imaginavam eutróficas.

Observou-se também que no sexo masculino eutrófico, $10 \%$ identificaram-se com magreza e apenas 3,33\% identificou-se com sobrepeso. Em relação aos que se encontravam com sobrepeso, observou-se o mesmo resultado das adolescentes do sexo feminino, na qual todos imaginavam-se eutróficos. Dos participantes classificados com obesidade, 66,6\% acreditavam estar acima do peso. De todos os adolescentes classificados com magreza, 71,43\% imaginavam-se eutróficos. As Tabelas 5 e 6 mostram a frequência de respostas quanto à percepção da imagem corporal desejada dos adolescentes. 
Tabela 5 - Frequência de respostas que retratam a autopercepção da imagem corporal (desejada) na escala de silhuetas (sexo feminino). Fortaleza-Ce, 2018.

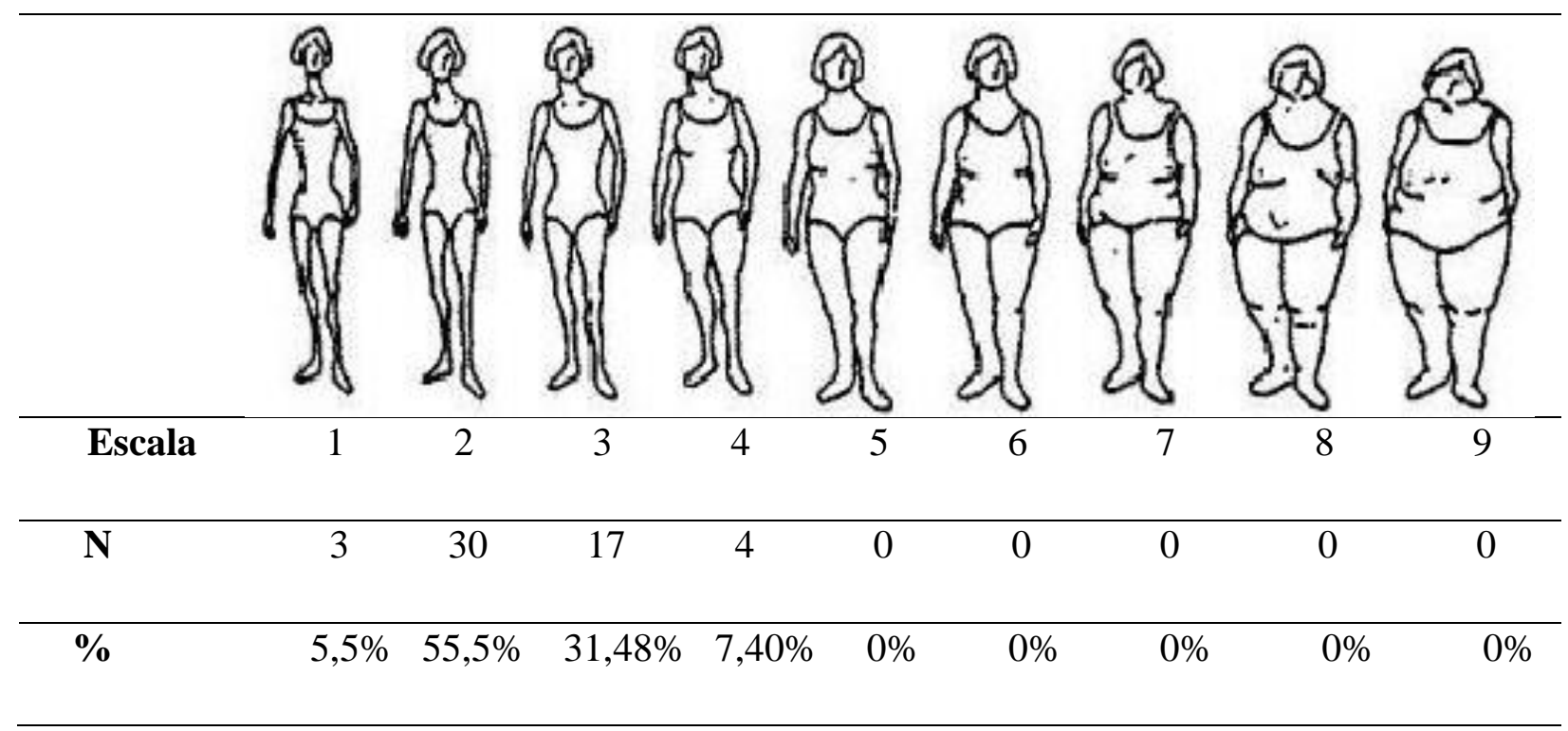

Fonte: Autores (2018).

Tabela 6 - Frequência de respostas que retratam a autopercepção da imagem corporal (desejada) na escala de silhuetas (sexo masculino). Fortaleza-Ce, 2018.

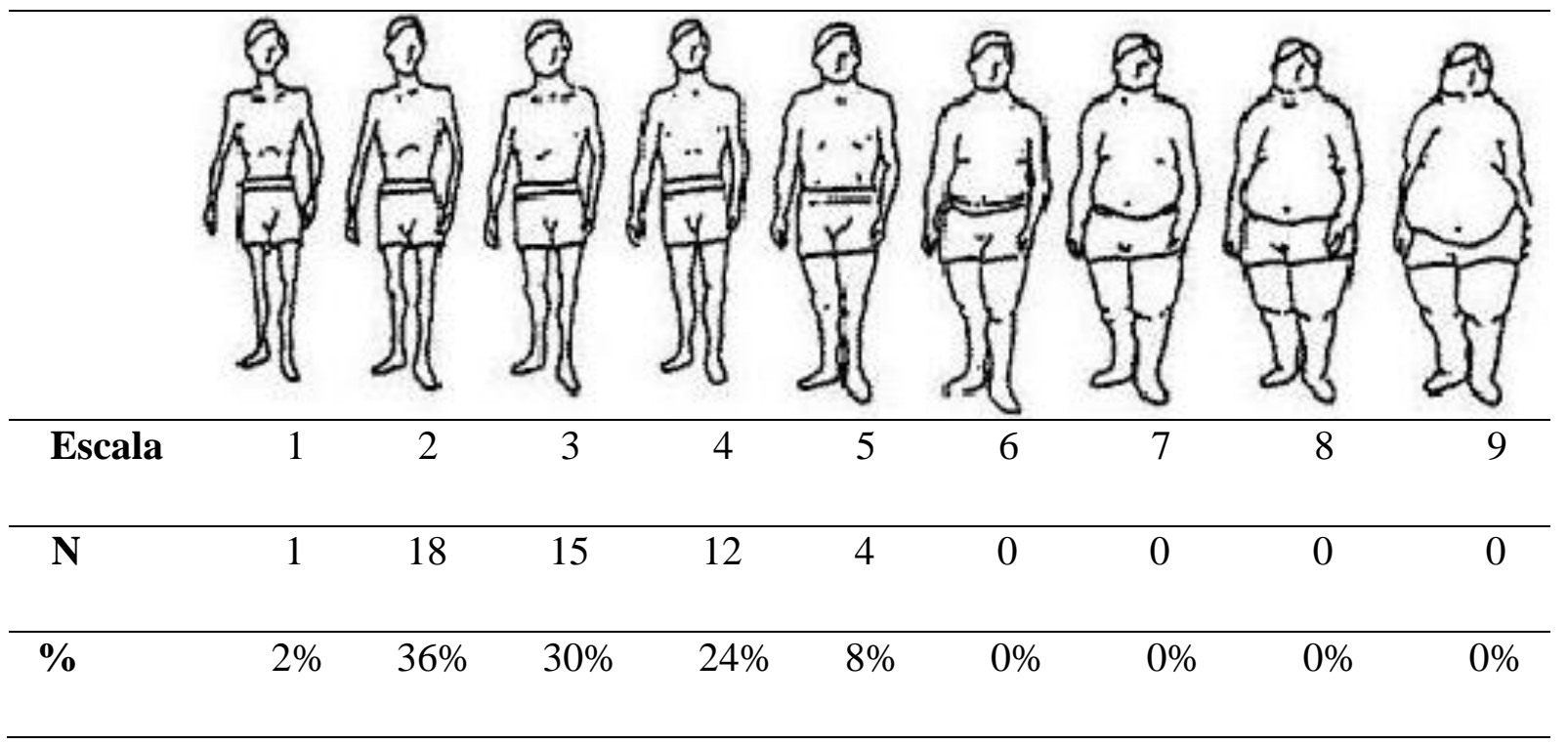

Fonte: Autores (2018).

Através da análise da Tabela 5, constatou-se uma frequência maior na silhueta de número 2 sendo a mais desejada entre o público feminino $(55,5 \%)$ e no sexo masculino (36\%) (Tabela 6). A Tabela 7 demonstra a quantidade de insatisfeitos e satisfeitos com a imagem corporal, de acordo com o sexo. A maior frequência de insatisfação (90\%) foi do sexo masculino e $(68,51 \%)$.no sexo feminino. 
Tabela 7 - Relação entre o sentimento de insatisfação e satisfação com a imagem corporal de adolescentes do ensino médio de uma escola pública da cidade de Fortaleza de acordo com o sexo. Fortaleza-Ce 2018.

\begin{tabular}{lc|c|c}
\hline \multirow{2}{*}{ Sexo } & \multicolumn{2}{c}{ Insatisfação e satisfação com a imagem corporal } \\
\cline { 2 - 4 } & $37(68,51 \%)$ & Satisfação N (\%) & Total N (\%) \\
\hline Feminino & $45(90 \%)$ & $17(31,48 \%)$ & $54(100 \%)$ \\
Masculino & $82(78,85 \%)$ & $5(10 \%)$ & $50(100 \%)$ \\
Total & & $22(21,15 \%)$ & $104(100 \%)$ \\
\hline
\end{tabular}

Fonte: Autores (2018).

\section{Discussão}

A imagem corporal é caracterizada pela forma como o sujeito se descreve e se sente sobre o próprio corpo, sendo uma interpretação mental multidimensional, pela qual ele se identifica e se julga com base na sua imagem física. Existem três componentes que são representados pelo conceito de imagem corporal, sendo o perceptivo, aquele que compreende a imagem do indivíduo, ou seja, peso, tamanho corporal e forma; o subjetivo, que está relacionado ao valor sentimental que o sujeito cria sobre sua imagem; e o comportamental que se refere a episódios que o sujeito se priva de se expor pela incomodidade com o seu corpo (Rocha et al., 2013).

Cecon (2017), em seu estudo realizado em Mato Grosso, com uma amostra de 2123 adolescentes de 10 a 19 anos, identificou que 56,9\% apresentavam distorção da imagem corporal, porém o presente estudo identificou que a maior parte dos adolescentes tem o conhecimento adequado da sua imagem. O mesmo estudo analisou a satisfação corporal e identificou que $79,3 \%$ dos adolescentes estavam insatisfeitos, corroborando com o presente estudo.

A insatisfação com a autoimagem tem sido reconhecida como problema crescente na população de adolescentes em diversos países, e o reconhecimento de tal fato enquanto questão de saúde pública é importante, devido aos riscos à saúde que predispõe (Martins, 2016). A insatisfação com o corpo refere-se à dimensão atitudinal da imagem corporal e corresponde a sentimentos negativos em relação ao peso e a forma do corpo, caracterizando essa variável com bastante significância na fase da adolescência (Fortes; Oliveira \& Ferreira, 2012).

No presente estudo foi identificado que ambos os sexos se encontravam insatisfeitos com a sua imagem corporal, na qual o sexo masculino foi o grupo que se encontrou com mais insatisfação. Fidelix e colaboradores (2011), em seu estudo realizado 405 adolescentes, de ambos os sexos, identificaram a prevalência de insatisfação com a imagem corporal de 56,5\%, sendo mais elevada no sexo masculino $(63,5 \%)$.

Pode-se afirmar que essa insatisfação é predominantemente elevada nos adolescentes, como no estudo realizado por Justino (2017) no estado de São Paulo, que avaliou 200 adolescentes dos sexos feminino e masculino, identificando que 72,7\% das meninas encontravam-se insatisfeitas com a sua imagem. Em contrapartida, a grande maioria dos meninos aproximadamente $80 \%$ estavam satisfeitos com a imagem corporal. Isso corrobora com o presente estudo referente à elevada taxa de insatisfação feminina, entretanto a de insatisfação do sexo masculino não foi confirmada.

A prevalência de insatisfação com a imagem corporal em ambos os sexos foi elevada. Resultado semelhante ao estudo de Alves et al. (2017), realizado no estado do Ceará, que ao analisarem 393 adolescentes do sexo feminino de 14 a 18 anos observaram que a maior parte $(65,4 \%)$ se encontravam insatisfeitos com a própria imagem. Essa prevalência elevada foi 
identificada pelo estudo de Carraro e colaboradores (2017), com uma amostra de 886 escolares, identificaram uma prevalência de insatisfação com a imagem corporal de $73 \%$ entre os adolescentes.

Essa alta frequência de insatisfação demonstrada no presente estudo corrobora com de Dias et al. (2017), no qual afirmam que a insatisfação com a imagem corporal na adolescência é mais prevalente, justificando que os adolescentes se tornam mais vulneráveis a sofrerem influências sobre padrões ideais de beleza, sendo a sociedade um dos pilares responsáveis para essa insatisfação.

Neste estudo observou-se que as escalas de silhuetas são bidimensionais, parecendo não caracterizar a imagem do indivíduo como um todo, podendo ser um fator limitante para identificação da percepção e insatisfação corporal. Vale ressaltar também que houve a limitação em relação à amostra dos adolescentes, pois muitos alunos não devolveram os termos assinados pelos pais, impossibilitando sua participação na pesquisa, o que resultou no tamanho menor da amostra.

\section{Considerações Finais}

A imagem corporal relacionada ao estado nutricional dos adolescentes apresentou uma autopercepção não condizente com seu estado nutricional real, porém isso não foi representado pela maior parte da amostra. Os adolescentes em sua maioria apresentaram uma subestimação do seu estado nutricional. Os resultados encontrados mostraram um percentual de insatisfação bastante representativo em ambos os sexos, sendo a classe masculina de maior insatisfação.

A insatisfação corporal é um fator preocupante que pode levar o adolescente a problemas psicológicos referentes à imagem, dentre eles a distorção da imagem corporal, classificado como um dos critérios de diagnóstico para os transtornos alimentares.

Dessa forma, ressalta-se a necessidade de desenvolver políticas públicas com o objetivo de combater o estereótipo de imagem ideal criada pela sociedade, bem como o desenvolvimento de movimentos sociais, com ênfase na educação nutricional e comportamental, realizada pelo profissional da saúde com o objetivo de diminuir tal incidência.

\section{Referências}

Alvarenga, M., Figueiredo, M., Fernanda, T., \& Antonaccio, C. (2015). Nutrição Comportamental. p.907, Barueri-sp: Manoele.

Alves, F. R., Bezerra, F. E. L., Sousa, E. A., \& Teixeira, F. A. A. (2017). Imagem corporal e fatores associados em meninas de escolas militares. Revista Brasileira em Promoção da Saúde, 30(4),1-7. https://doi.org/10.5020/18061230.2017.6082

Brasil. Conselho Nacional de Saúde. Resolução $\mathrm{N}^{\mathrm{o}}$ 466, de 12 de dezembro de 2012. https://www.inca.gov.br/sites/ufu.sti.inca.local/files// media/document//resolucao-cns-466-12.pdf

Branco, L. M., Hilário, M. O. E. \& Cintra, I. D. P. (2006). Percepção e satisfação corporal em adolescentes e a relação com seu estado nutricional. Archives of Clinical Psychiatry, 33(6), 292-296. https://doi.org/10.1590/S1413-81232012000900030

Carvalho, L. C. D. (2019). A Influência das mídias sociais na imagem corporal e no desenvolvimento de transtornos alimentares em estudantes do sexo feminino da Faculdade Pernambucana de Saúde do Recife-Pe (Trabalho de Conclusão de Curso). Faculdade Pernambucana de Saúde, Brasil. https://tcc.fps.edu.br/handle/fpsrepo/616

Carraro, F., Rech, R. R., Frata, B., Halpem, R., Zanol, F., Colognese, A. R. et al., (2017). Insatisfação com a imagem corporal em adolescentes na cidade de Farroupilha, RS. Revista Associação Médica Rio Grande do Sul, 61(1),10-13. https://pt.calameo.com/read/0007562466cb5aa5e5a3d

Cecon, R. S. (2017). Indicadores Cardiometabólicos, densidade mineral óssea e percepção da imagem corporal de adolescentes de 10 a 19 anos, com e sem triagem positiva para transtornos alimentares, do município de Viçosa - MG. Tese de Doutorado - Universidade Federal de Viçosa. https://www.locus.ufv.br/handle/123456789/12906

Dias, N. (2017). Percepção da imagem corporal de adolescentes com deficiência visual: associação com prática de atividade física e participação nas aulas de educação física. Trabalho de conclusão de curso (licenciada em Educação Física) - Universidade Federal de Santa Catarina. Florianópolis, p.11. https://repositorio.ufsc.br/handle/123456789/184765

Fidelix, Y. L., Silva, D. A. S., Pelegrini, A., Silva, A.F. \& Petrosky, E. L. (2011). Insatisfação com a imagem corporal em adolescentes de uma cidade de pequeno porte: associação com sexo, idade e zona de domicílio. Revista Brasileira de Cineantropometria Desempenho Humano. 12(3),202-207. https://doi.org/10.5007/1980-0037.2011v13n3p202 
Research, Society and Development, v. 10, n. 1, e8710111484, 2021 (CC BY 4.0) | ISSN 2525-3409 | DOI: http://dx.doi.org/10.33448/rsd-v10i1.11484

Fontenele, R. M., Ramos, A. S. M. B., Goiabeira, C. R. F., Cutrim, D. S., Galvão, A. P. F. C., \& Noronha, F. M. F. (2019). Impacto dos transtornos alimentares na adolescência: uma revisão integrativa sobre a anorexia nervosa. Revista Enfermagem Atual InDerme,87(25),1-9. https://doi.org/10.31011/reaid-2019-v.87-n.25-art.201

Fortes, L. S., Almeida, S. S. \& Ferreira. M. E. C. (2013). Imagem corporal e transtornos alimentares em atletas adolescentes: Uma revisão. Psicologia em estudo,18(4),667-677. https://www.scielo.br/pdf/pe/v18n4/09.pdf

Fortes, L. S., Oliveira, F. G. \& Ferreira, M. E. C. (2012). Influência de fatores afetivos, antropométricos e sociodemográficos sobre o comportamento alimentar em jovens atletas. Jornal Brasileiro de Psiquiatria, 61(3), 148-153. https://doi.org/10.1590/S0047-20852012000300005

Gomes, T. L. M. (2015). Percepção da autoimagem em escolares do ensino médio na rede federal de educação e tecnologia. Dissertação (Dissertação em saúde pública e meio ambiente) São Luis- MA, p.10. https://www.arca.fiocruz.br/handle/icict/14207

Justino, M. I. C. (2017). Autopercepção da imagem e satisfação corporal e estado nutricional de adolescentes. Dissertação de mestrado (Dissertação em Ciências da Saúde). Campinas - SP, 47. http://tede.bibliotecadigital.puccampinas.edu.br:8080/jspui/handle/tede/1023

Lopes, M. S., Mendes, R. C. P. R. \& Sousa, S. M. N. (2017). Ser mulher: Uma análise da imagem corporal entre adolescentes. Revista Espacios, 38(29)1-9. https://www.revistaespacios.com/a17v38n29/a17v38n29p03.pdf

Madrigal-fritsch, H., Irala-estevez, J., Martinez-gonzalez, M. A., Kearney, J., Gibney, M., \& Martinez-hernandez, J. A. (1999). Percepción de la imagen corporal como aproximación cualitativa al estado de nutrición. Salud pública de México, 41(6)479-486. e https://core.ac.uk/download/pdf/83579632.pdf

Martins, C. R. Efetividade de uma intervenção educacional na imagem corporal de adolescentes. (2016.) Tese (Doutorado) - Curso de Educação Física, Centro de Desportos, Universidade Federal de Santa Catarina, Florianópolis. p.214. https://repositorio.ufsc.br/bitstream/handl e/123456789/174887/345355.pdf?sequence=1\&isAllowed=y

Martins, C. R, Pelegrine, A., Matheus, S. C. \& Petroski, E. L. (2010). Insatisfação com a imagem corporal e relação com estado nutricional, adiposidade corporal e sintomas de anorexia e bulimia em adolescentes. Rev Psiquiatr Rio Gd Sul, 32(1)19-23. https://doi.org/10.1590/S0101-81082010000100004

Rocha, M., Barlhichoto, M., Lopes, J., Costa, K. \& Nacif, M. (2013). Vigorexia: um distúrbio da imagem corporal. Revista Digital Buenos Aires, 18(181).

SANTOS, G. E. O. (2018). Cálculo amostral: calculadora on-line. http://www.calculoamostral.vai.la

Silva, S. M. C. S., Mura, J. D. P. (2016). Tratado de alimentação, nutrição \& dietoterapia. p.842, (3a ed.), Roca.

Stunkard A. J., Sorensen T. \& Schulsinger F. (1983). Use of the Daniel Adoption Registry for the study of obesity and thinness. In: Skety, S. et al. Genetics of neurological and psychiatric disorders. 115-120. New York.

World Health Organization. WHO: Growth reference data for 5-19 years (2007). http://www.who.int/growthref. 